\title{
Effets de la production de charbon de bois sur les fourrés xérophiles, cas du plateau de Belomotse, Madagascar
}

Josoa R. Randriamalala', „, Radobarimanjaka Rabenialal and Haja N. Masezamanal

\author{
Correspondence: \\ Josoa R. Randriamalala \\ Diagnostic Environnemental et Recherche Appliquée pour le \\ Développement en Milieu Rural (DERAD) \\ Lot AKT II A 105, Ambohitsaratelo, Vontovorona \\ BP 60115 \\ Antananarivo 102 \\ Madagascar \\ Email: rramarolanonana@yahoo.fr
}

\begin{abstract}
Woody charcoal production is the main cause of dry forest degradation in the southwestern Madagascar. This paper analyses the effects of this practice on the diversity, represented by species richness, structure, species composition and regeneration of the xerophytic thickets on the Belomotse Plateau, Toliara II District. Eleven $400 \mathrm{~m}^{2}$ plots were randomly sampled in woody charcoal production sites and 10 in control sites. Woody charcoal production has reduced xerophytic thickets mean height, density and mean diameter at breast height. It has also affected species composition while it has not affected xerophytic thicket diversity or its regeneration. However, its regeneration rate is low on both woody charcoal production and control sites. Decline of pollinator insect communities or exogenous causes such as increasing climate aridification or escalation of rainfall variability may explain this low regeneration rate. Woody charcoal production in semi arid region is an unsustainable activity.
\end{abstract}

\section{RÉSUMÉ}

La production de charbon de bois est l'une des causes de la dégradation des forêts sèches du Sud-Ouest malgache. Cet article analyse les effets de cette pratique sur (i) la diversité biologique représentée par la richesse spécifique, (ii) la structure et (iii) la composition floristique des fourrés xérophiles du plateau de Belomotse, Toliara II. Onze placeaux de $400 \mathrm{~m}^{2}$ ont été sélectionnés de manière aléatoire dans des sites de production de charbon de bois et 10 dans des sites témoins. La production de charbon de bois affecte la composition floristique des fourrés xérophiles. De plus, elle réduit leur hauteur, densité et diamètre moyen à hauteur de poitrine (dhp). En revanche, elle n'affecte pas la richesse spécifique ni la régénération naturelle. Les taux de régénération sont cependant faibles, indiquant un mauvais recrutement au sein des peuplements végétaux qui occupent les fourrés xérophiles. Le dépérissement des insectes pollinisateurs, ou des causes exogènes telles que l'aridification croissante et/ou l'accentuation de la variabilité interannuelle des précipitations peuvent expliquer cette mauvaise régénération. La production de charbon de bois en zone semi-aride n'est pas une activité durable.

\section{INTRODUCTION}

Le charbon de bois est une source d'énergie utilisée essentiellement pour la cuisson en Afrique et pour des usages industriels en Amérique latine (Chidumayo et Gumbo 2013). Son caractère accessible et le coût prohibitif des autres sources d'énergie (ex. gaz, électricité) contribuent au maintien de son usage dans les pays pauvres. Les études sur le charbon de bois se focalisent essentiellement sur l'aspect socio-économique de sa production (Zulu 2010, Minten et al. 2013, Sander et al. 2013). L'analyse des effets de cette activité sur le fonctionnement des écosystèmes forestiers est rare (Chidumayo et Gumbo 2013). Cette activité réduit la densité forestière et la biomasse (Kouami et al. 2009, Chidumayo et Gumbo 2013).

Une grande partie des forêts sèches malgaches a été détruite et peu de grands blocs subsistent (Seddon et al. 2000, Blanc-Pamard et al. 2005). L'agriculture itinérante sur brûlis et la fabrication de charbon de bois sont les principales causes de cette déforestation et/ou dégradation des forêts (Casse et al. 2004, Blanc-Pamard et al. 2005). La production de charbon de bois touche particulièrement les fourrés xérophiles qui occupent la partie côtière du Sud-Ouest malgache (Raoliarivelo et al. 2010), la zone la plus aride de Madagascar. En effet, ces fourrés xérophiles fournissent la quasi totalité des bois exploités pour la production 
de charbon de bois consommé dans la ville de Toliara. La demande de cette ville en charbon de bois augmente avec sa population (Gardner et al. 2015) et accentue davantage encore les pressions exercées sur les fourrés xérophiles des alentours dans un rayon de $40 \mathrm{~km}$ (Masezamana et al. 2013, Rabeniala et al. 2013). Ces fourrés xérophiles sont des formations arbustives caractérisées par la dominance de Didieraceae et Euphobiaceae et présentent un taux d'endémisme floristique élevé (Koechlin et al. 1997).

Les interactions entre la production de charbon de bois et le pâturage caprin, d'une part, et les fourrés xérophiles du SudOuest malgache, d'autre part, ont déjà été analysées (Rabeniala et al. 2009, Raoliarivelo et al. 2010, Randriamalala et al. 2016). II a notamment été montré que la combinaison de ces pratiques (charbon et pâturage caprin) réduit la biomasse ligneuse et modifie la composition floristique des fourrés xérophiles sur sable roux (Randriamalala et al. 2016). En revanche, le pâturage caprin seul n'affecte ni la diversité ni la structure des fourrés sur dalle calcaire même si cette formation végétale se régénère mal (Randriamalala et al. 2016) et a une faible productivité en biomasse (Randriamalala et al. 2015). On en sait donc peu sur les effets de la production de charbon de bois sur les fourrés xérophiles sur dalle calcaire, le type de sols prédominants dans les plateaux Mahafaly et de Belomotse du Sud-Ouest malgache.

Ce papier analyse les effets de la fabrication de charbon de bois sur la diversité, la structure et la régénération naturelle des fourrés xérophiles sur dalle calcaire du Sud-Ouest malgache.

\section{MATÉRIELS ET MÉTHODES}

SITE D'ÉTUDE. Le site d'étude est localisé sur le plateau de Belomotse (communes d'Andranohinaly, d'Ambohimaha-veIona, de Mihary et de Behompy, District de Toliara II, Figure 1). Le climat est semi-aride avec des précipitations annuelles moyennes de $418 \mathrm{~mm}$ pour la station de Toliara (Rabeniala et al. 2009). La végétation naturelle est formée par des fourrés xérophiles à Didieraceae et à Euphorbia spp. (Radosy 2013). Les deux principaux types de sol sont des dalles calcaires et des sables roux (Rabeniala et al. 2013). Les principales activités des populations locales sont l'agriculture qui se concentre dans les plaines alluviales des fleuves Fiherenana au nord et Onilahy au sud (Figure 1), l'élevage de petits ruminants et la fabrication de charbon de bois qui supplée à I'insuffisance des revenus agricoles et d'élevage (Masezamana et al. 2013). L'agriculture sur brûlis est également pratiquée dans les fourrés xérophiles sur le plateau (Rabeniala et al. 2013).

ACTIVITÉ DE PRODUCTION DE CHARBON DE BOIS SUR LE PLATEAU DE BELOMOTSE. Les sites de production de charbon de bois se concentrent autour de villages tels qu'Ankiliberengy, Ampamata, Ankazomena et Ambohimahavelona et autour de campements permanents tels que celui de Betsinefo (Figure 1). Ces campements sont occupés par des gens marginaux qui ont fait de la prison à Toliara ou qui ne possèdent pas de terrain dans les plaines fertiles alluviales depuis environ huit ans (Rabeniala et al. 2013). Les activités principales des occupants de tels campements sont la production de charbon de bois et la collecte de bois de feu. Les techniques de production de charbon de bois y sont les mêmes que celles décrites par Randriamalala et al. (2016). La différence réside dans le fait que les fours à charbon sur le plateau de Belomotse sont essentiellement creusés dans des sols calcaires rocailleux (dalle calcaire) contrairement à ce qui
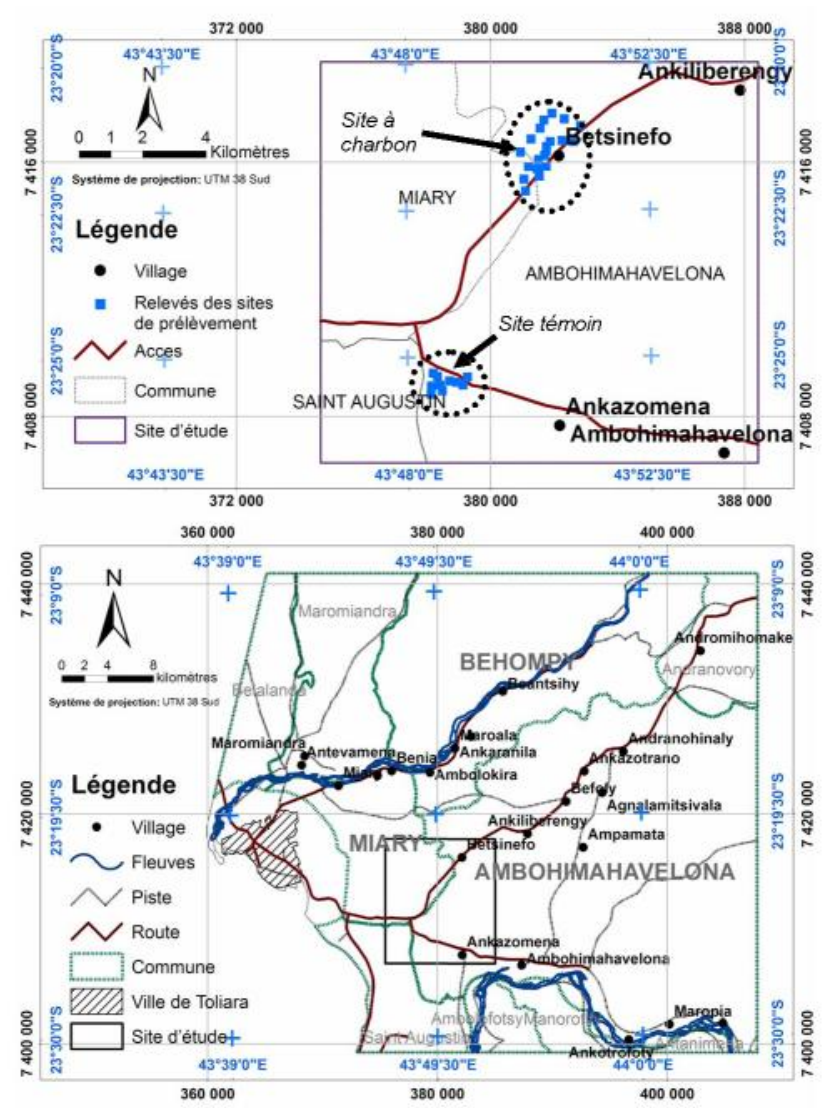

Figure 1. Localisation des sites d'étude.

se passe à Soalara Sud, où les fours à charbon sont exclusivement installés sur sable roux (Randriamalala et al. 2016). Les roches calcaires de taille inférieure $(<20-30 \mathrm{~cm})$ sont mises de côté et le four à charbon est creusé dans les sols meubles sousjacents et entre les dalles calcaires. Les sols à sables roux sont généralement exploités pour l'agriculture (Rabeniala et al. 2013, Randriarimalala 2016).

La quasi-totalité de la production de charbon de bois est vendue à des intermédiaires qui les transportent par camion vers la ville de Toliara (Masezamana et al. 2013) même si une faible proportion est transportée par bicyclette vers la même ville par les producteurs eux même (Obs. pers.).

RELEVÉS FLORISTIQUES. Onze placeaux de $400 \mathrm{~m}^{2}$ ont été sélectionnés aléatoirement dans les sites de production proches du campement permanent de Betsinefo, appelés sites à charbon de bois et 10 l'ont été dans des sites, plus au sud, à plus de $3 \mathrm{~km}$ à vol d'oiseau de tout village et/ou campement permanent (Figure 1). Ces derniers se trouvent dans des sites moins perturbés, qualifiés de témoins. En effet, les principaux villages le long de la Route Nationale 7 se trouvent au nord, du village d'Ankiliberengy et du campement de Betsinefo (Befoly, Ankazotrano, Andranohinaly, et Andranovory, Figure 1). Les autres villages importants se trouvent au sud-est (vers la route d'Ambohimahavelona, Figure 1). Les sites actuels de production de charbon de bois se trouvent essentiellement entre les villages d'Ambohimahavelona et d'Ankiliberengy (au nord-est des sites témoins, Rabeniala et al. 2013). Par conséquent, les sites les moins perturbés se trouvent plus au sud (Figure 1). Peu de fours à charbon abandonnés ont été observés dans ces sites témoins contrairement aux sites proches de Betsinefo où la densité de tels fours 
est plus importante. Tous les placeaux sélectionnés se trouvent sur dalle calcaire, le type de sol prédominant.

Les sites à charbon de bois et les sites témoins ne sont pas pâturés par des caprins, ni par des zébus. Le site d'étude est un campement permanent et ses occupants sont des gens marginaux qui exploitent les fourrés xérophiles pour le bois (bois de feu, gaulette et surtout charbon de bois) et accessoirement pour l'agriculture (hatsake). Les éleveurs les plus proches se trouvent dans le village d'Ankiliberengy, à plus de $5 \mathrm{~km}$ à vol d'oiseau. Or il a été montré dans des cas similaires (à Soalara-Sud) que les pâturages caprins s'étendaient rarement à plus de $4 \mathrm{~km}$ autour des enclos (Rabeniala et al. 2009, Randriamalala 2014, Randriamalala et al. 2016).

Les arbres et arbustes de plus de 1,3 m de hauteur appartenant à la strate supérieure ont été inventoriés dans la surface de $400 \mathrm{~m}^{2}$ partagée en quatre placettes de $100 \mathrm{~m}^{2}$. Ensuite, une des placettes de $100 \mathrm{~m}^{2}$ a été choisie au hasard pour l'étude de la régénération consistant à inventorier tous les individus de moins de 1,3 m, qui appartiennent à la strate inférieure. Les paramètres de relevé dans le placeau ont permis de calculer les indices de diversité et de structure suivants: la richesse spécifique de la strate supérieure, qui est le nombre d'espèces dans le placeau de $400 \mathrm{~m}^{2}$, la densité d'arbustes dans la strate supérieure, le dhp moyen des individus appartenant à la strate supérieure (le diamètre de la plus grosse tige a été mesuré si l'arbuste considéré présente des ramifications à moins de 1,3 $\mathrm{m}$ du sol), la hauteur maximale de la strate supérieure, la richesse spécifique de la strate inférieure qui est le nombre d'espèces dans la placette de $100 \mathrm{~m}^{2}$ et la densité d'arbustes dans la strate inférieure. Les taux de régénération (TR) des espèces arbustives ont également été calculés comme suit : $T_{\mathrm{ij}}=\mathrm{n}_{\mathrm{ij}} / \mathrm{N}_{\mathrm{ij}}$, avec $\mathrm{TR}_{\mathrm{ij}}$ taux de régénération de l'espèce i dans le placeau j de $400 \mathrm{~m}^{2}, \mathrm{n}_{\mathrm{ij}}$, nombre d'individus appartenant à la strate inférieure ( $<1,3 \mathrm{~m}$ de haut) et à l'espèce i dans le carré de $100 \mathrm{~m}^{2}$ du placeau j, $\mathrm{N}_{\mathrm{ij},}$ nombre d'individus de l'espèce i appartenant à la strate supérieure ( $\geq 1,3 \mathrm{~m}$ de haut) dans le même carré de $100 \mathrm{~m}^{2}$. La moyenne des taux de régénération par traitement a également été calculée : $T R=\Sigma\left(a_{i} / A\right) \times T R_{i}(i=1$ $a_{\mathrm{N}}{ }_{\mathrm{N}}$, avec $\mathrm{a}_{i}$, répétition de l'espèce i dans le traitement considéré, $A$, nombre d'espèces dans le même traitement, $T_{\mathrm{i}}$, taux de régénération moyen de l'espèce i dans le même traitement.

ANALYSE DES DONNÉES. Des analyses factorielles des correspondances (AFC, XIstat 6.03, Addinsoft 1995-2008) ont été appliquées aux données portant sur les espèces des placeaux de $400 \mathrm{~m}^{2}$ pour analyser les effets de la production de charbon de bois sur la composition floristique de la strate supérieure des fourrés xérophiles. Des tests de comparaisons de moyennes (XIstat 6.03) ont également été appliqués sur les variables de diversité et de structure pour analyser leurs variations par rapport aux perturbations liées au charbon de bois.

Des tests en deux parties (two-part tests, TPT, Lachenbruch 2001) ont été effectués pour analyser les variations du taux de régénération par rapport aux perturbations liées au charbon de bois. En effet, la moyenne n'est pas une synthèse satisfaisante des données relatives au taux de régénération puisqu'elles présentent beaucoup de zéros (45-59\%). Le TPT est une combinaison de tests qui prend en compte le fait que la somme de deux statistiques suivant une distribution 2 suit une distribution 2 avec un degré de liberté (ddl) égale à la somme des ddl des deux statistiques considérées (Delucchi et Bostrom 2004). L'hypothèse nulle est l'égalité des proportions de zéros dans les $k$ échantillons $(k \geq 2)$ et celle des moyennes des parties non nulles des $k$ échantillons. Pour nos données, un test de fréquences pour deux échantillons (TPZ, XIstat 6.03) avec un ddl a été utilisé pour comparer les proportions de zéros et un test de Kolmogorov-Smirnov (KS, XIstat 6.03) a été utilisé pour comparer les moyennes des valeurs non nulles (Lachenbruch 2001, Delucchi et Bostrom 2004). La statistique du TPT est donnée par la relation : TPS $=Z^{2}+W^{2}$, Z est la statistique du TPZ et W celle de KS et TPS suit une loi du 2 avec deux ddl.

\section{RÉSULTATS}

COMPOSITION FLORISTIQUE. Le premier axe de I'AFC

(18,69\% de l'inertie totale, Figure 2) ne correspond à aucun gradient particulier. Le second axe (14,84\%) sépare les placeaux des sites à charbon de bois des sites témoins (Figure 2). La végétation des sites témoins est caractérisée par Chadsia grevei (6\% de la valeur propre du second axe factoriel), Commiphora lamii $(5,6 \%)$, Euphorbia leucodendron (4\%), Ruellia albopurpurea (3,5\%), Tetrapterocarpon geayi (3,4 \%), Bauhinia grandidieri (1 \%), Commiphora sp. (3\%), Operculicarya sp. (2,8\%), Dicraeopetalum mahafaliense $(0,9 \%)$. Des arbustes à bois dur tels que $C$. lamii, $R$. albopurpurea, B. grandidier et $D$. mahafaliense caractérisent le site témoin. La végétation des sites à charbon de bois est caractérisée par Grewia tulearensis (9,3\%), Cassia meridionalis (4,6 \%), Mimosa delicatula (3,7 \%), Ruellia sp. (3,7 \%), Vaughania interrupta (3,3\%), Dichrostachys alluaudiana $(2,6 \%)$, Commiphora marchandii (2,1\%), Pristimera bojeri (1,8\%), Euphorbia dutso (1,7\%), Paederia grandidieri $(1,4 \%)$, Cynanchum sp. $(1,2 \%)$, Grewia calvata $(1,2 \%)$, Ormocarpum bernierianum $(1,1 \%)$ et Croton geayi $(1,1 \%)$. Le site à charbon est essentiellement caractérisé par des lianes (P. grandidieri et Cynanchum sp.) ainsi que par des arbustes de
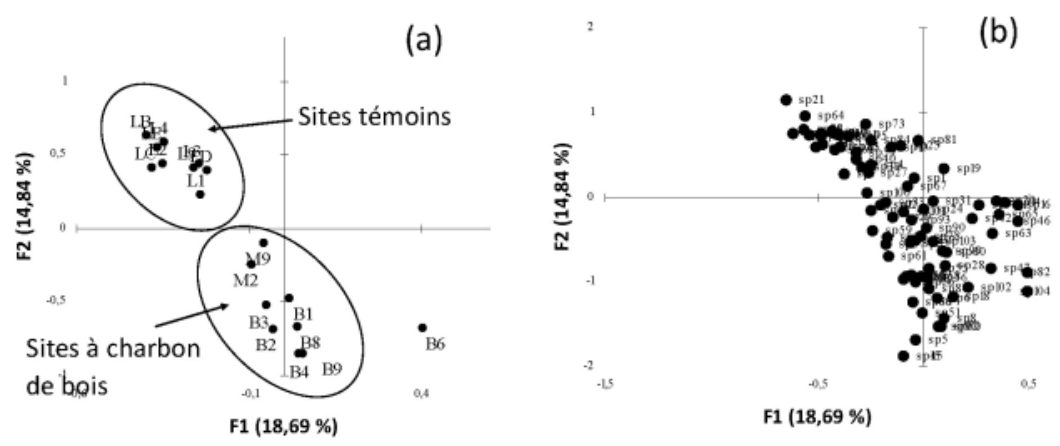

Figure 2. Projections des placeaux (a) et des espèces des strates supérieures $(\geq 1,3 \mathrm{~m} ; \mathrm{b})$ dans le premier plan factoriel de I'AFC. 
petite taille ( $<2 \mathrm{~m}$ de haut, $O$. bernierianum et $C$. geayi) et par des arbustes à bois relativement tendre (G. tulearensis, C. meridionali et C. marchandii). Des espèces à bois dur telles que Diospyros manampetsae H. Perrier, Neobeguea mahafalensis Leroy, Securinega perrieri Leandri et Cedrelopsis grevei Baillon sont communes aux deux sites. La production de charbon de bois affecte la composition floristique des fourrés xérophiles.

DIVERSITÉ, STRUCTURE. Les richesses spécifiques moyennes associées à la strate supérieure des fourrés xérophiles ne varient pas significativement avec les sites considérés ( $t=0,497$, $p>0,05$, Tableau 1). En revanche, les moyennes des hauteurs maximales, des diamètres moyens et celles des densités varient significativement ( $p<0,05$, Tableau 1). La production de charbon de bois n'affecte pas la richesse spécifique des fourrés xérophiles mais affecte leurs structures : cette activité engendre une baisse significative de la hauteur, du diamètre et de la densité. En ce qui concerne la strate inférieure, une variation significative de la richesse spécifique moyenne a été constatée par rapport aux sites à charbon de bois $(t=3,484, p<0,01$, Tableau 1). En moyenne, la strate inférieure des fourrés xérophiles dans les sites à charbon de bois présente une richesse spécifique plus importante que celles des fourrés xérophiles dans les sites témoins. En revanche, les moyennes des densités d'arbustes n'ont pas varié de façon significative avec les sites ( $t=0,520, p>0,05$, Tableau 1$)$. Les taux de régénérations moyens ne varient pas significativement avec les sites considérés ( $2=2,214, p>0,05$, Tableau 1). Les activités de fabrication de charbon de bois n'affectent pas la régénération naturelle des fourrés xérophiles. En revanche, cette régénération est mauvaise car la moitié des espèces arbustives recensées ont des taux de régénération de moins de $10 \%$ (Tableau 1).

\section{DISCUSSION}

LA PRODUCTION DE CHARBON DE BOIS AFFECTE LA COMPOSITION FLORISTIQUE DES FOURRÉS XÉROPHILES.

L'AFC effectué sur les individus de la strate supérieure a permis de séparer la composition floristique des sites de production de celle des sites témoins. Ces résultats confirment ceux de Randriamalala et al. (2016) qui ont montré que la production de charbon de bois affectait la composition floristique de fourrés xérophiles sur sable roux à Soalara-Sud, à une cinquantaine de kilomètres au sud du site d'étude. Ces résultats sont également en accord avec ceux de Colón et Lugo (2006), dans une zone semiaride du Costa Rica avec des précipitations moyennes annuelles de $860 \mathrm{~mm}$. Ces chercheurs ont montré, en comparant des indices de similarité, que la différence entre la composition floristique d'anciens sites de production de charbon de bois et celle de forêts matures adjacentes persiste plusieurs années après

Tableau 1. Variations des paramètres de diversité et de structure. $n$ : Répétitions : $\mathrm{S}$ : Richesse spécifique de la strate supérieure $\left(.400 \mathrm{~m}^{-2}\right) ; \mathrm{Hm}$ : Hauteur moyenne (m) ; DHPm : Diamètre moyen à $1,3 \mathrm{~m}(\mathrm{~cm})$; D : Densité d'arbustes de la strate supérieure $\left(.400 \mathrm{~m}^{-2}\right) ; \mathrm{S}^{\prime}$ : Richesse spécifique de la strate inférieure $\left(.100 \mathrm{~m}^{-2}\right)$;

$D^{\prime}$ : Densité d'arbustes de la strate inférieure $\left(.100 \mathrm{~m}^{-2}\right)$; TR : Taux de régénération moyen par traitement (\%), nombres entre parenthèses représentent respectivement : premier quartile ; médiane ; troisième quartile, en \% ; $p$ : Degré de signification des tests de comparaison de moyennes.

\begin{tabular}{lccl} 
Sites & Sites à charbon de bois & Sites témoins & $p$ \\
\hline n & 11 & 10 & \\
S & 34.73 & 36.1 & $>0,05$ \\
Hm & 1.89 & 2.23 & $<0,01$ \\
DHPm & 1.36 & 2.18 & $<0,01$ \\
D & 207.18 & 269.1 & $<0,05$ \\
S' $^{\prime}$ & 12.82 & 8.1 & $<0,01$ \\
D $^{\prime}$ & 43.91 & 39.5 & $>0,05$ \\
TR & $59(0 ; 9 ; 55)$ & $63(0 ; 0 ; 48)$ & $>0,05$
\end{tabular}

I'abandon de cette activité. La production de charbon de bois marque les fourrés xérophiles par le prélèvement des espèces à bois dure et épargne les espèces à bois tendre (Raoliarivelo et al. 2010, Ramaroson 2014).

LA PRODUCTION DE CHARBON DE BOIS N'AFFECTE PAS LA DIVERSITÉ DES FOURRÉS XÉROPHILES. La diversité qui est représentée par la richesse spécifique n'est pas affectée par la production de charbon de bois. En effet, la variation, au cours du temps, de la richesse spécifique d'une formation végétale est le produit de (i) l'arrivée de nouvelles espèces par la germination de graines de (ii) la mortalité d'individus appartenant aux espèces résidente. La combinaison (i) de la variation de la composition floristique et (ii) de l'absence d'une différence significative entre les richesses spécifiques moyennes par rapport à la production de charbon de bois semble montrer que cette activité favorise la mortalité d'espèces à bois dure et l'émergence de nouvelles espèces que cette même activité épargne. Ces dernières peuvent être des arbustes de petite taille tels que $O$. bernierianum et C. geayi. A termes, la production de charbon de bois risque de mener à une extinction localisée (dans les sites de production) des espèces à bois dure. De plus, la variation de la composition floristique et la constance de variables telles que la richesse spécifique et la productivité sont souvent observées quand un écosystème subit des perturbations (Briske et al. 2003). II a même été supposé que cette fluctuation d'espèces (bilan quasi-nul entre perte et gain) est un mécanisme de compensation qui contribue à la stabilité de l'écosystème (homéostasie, Ernest et Brown 2001).

Nos résultats sont en accord avec ceux de Randriamalala et al. (2016) qui ont montré que la production de charbon de bois et le pâturage caprin n'affectent pas la richesse spécifique des fourrés xérophiles sur sable roux à Soalara-Sud. Ces résultats sont par contre en désaccord avec ceux de Kouami et al. (2009) au Togo $\left(\mathrm{P}=800-1500 \mathrm{~mm}^{\mathrm{an}}{ }^{-1}\right)$ qui ont montré que la production de charbon de bois occasionne généralement une baisse significative du nombre d'espèces ligneuses. Par contre, Colon et Lugo (2006) ont montré qu'au Costa-Rica ( $\mathrm{P}=860 \mathrm{~mm}$ an-1), les recrus se développant sur d'anciens sites de production de charbon de bois sont plus riches en espèces ligneuses que les forêts matures et les recrus post-agricoles adjacents. La régénération des sites de production de charbon de bois est donc possible. L'interaction richesse spécifique de la végétation-production de charbon de bois ne semble pas obéir à des schémas uniformes. Les pratiques de la production de charbon de bois, c'est à dire le caractère sélectif ou non (Chudimayo et Gumbo 2013) et l'intensité des prélèvements de bois peuvent expliquer ces différences. Ces pratiques peuvent perturber à des degrés différents les potentiels de régénération des forêts concernées (souches en place, germination de graines provenant de la banque et/ou de la pluie de graines) qui s'expriment différemment.

LA PRODUCTION DE CHARBON DE BOIS AFFECTE LA STRUCTURE DES FOURRÉS. La production de charbon de bois réduit la hauteur maximale, la densité des arbustes et le dhp des fourrés xérophiles. Ces résultats confirment ceux de Randriamalala et al. (2016) qui ont montré que le pâturage caprin et la production de charbon de bois réduisent les hauteurs moyennes, la densité d'arbustes et la biomasse aérienne des fourrés xérophiles sur sable roux à Soalara-Sud. De même, Kouami et al. (2009) au Togo ont trouvé que les végétations des sites exploités 
pour le charbon de bois présentent généralement des hauteurs moyennes, des densités et des dhp plus faibles que celles des sites intacts.

La baisse de la densité et du dhp semble indiquer que la productivité en biomasse ligneuse des fourrés xérophiles serait inférieure au prélèvement de bois occasionné par la production de charbon de bois. En effet, la biomasse aérienne des formations arbustives est en général, positivement corrélée avec le dhp (Navar et al. 2004, Ali et al. 2015). Cette activité n'est donc pas durable et peut aboutir à termes à une extinction localisée des espèces à bois dure, propices à la fabrication de charbon de bois (Ramaroson 2014). En effet, la lenteur de la croissance des espèces arbustives des fourrés xérophiles est telle qu'il faut une rotation de plus de 55 ans pour produire la biomasse actuelle (Randriamalala et al. 2015).

UNE MAUVAISE RÉGÉNÉRATION DES FOURRÉS XÉROPHILES.

La production de charbon de bois favorise la diversité de la végétation de la strate inférieure, mais n'affecte pas de façon significative leurs densités, même si celle des sites à charbon semble être plus importante. Ces résultats vont en partie à l'encontre de ceux de Randriamalala et al. (2016) qui ont trouvé que la production de charbon de bois et le pâturage caprin n'affectaient pas la richesse spécifique de la strate inférieure des fourrés xérophiles sur sable roux à Soalara-Sud. La production de charbon de bois contribue à créer des trouées qui sont des moteurs de dynamiques forestières en zones humides mais dont l'importance pour la régénération des forêts sèches reste à démontrer (Quesada et al. 2009). Quoiqu'il en soit, il a été constaté que la régénération des forêts sèches ayant été exploité pour le charbon de bois est possible (Colón et Lugo 2006) et la diversité de la strate inférieure des végétations des sites exploités est cohérente avec cette théorie.

Les fourrés xérophiles se régénèrent cependant mal. Les taux de régénération des espèces qui s'y trouvent dépassent rarement les $100 \%$; c'est-à-dire que l'abondance des individus de régénération d'une espèce donnée est rarement plus importante que celle des individus matures semenciers appartenant à la même espèce. Le peuplement végétal dans les fourrés xérophiles est vieillissant : il n'y a pas suffisamment d'individus de régénération pour remplacer ceux qui sont matures quand ces derniers meurent. Cette mauvaise régénération a également été observée dans les fourrés xérophiles du plateau Mahafaly à une cinquantaine de kilomètres au sud du site d'étude (taux moyen de régénération de 44-235\%, Randriamalala et al., 2016). Cette mauvaise régénération n'est pas imputable à la production de charbon de bois puisqu'elle s'observe même dans les sites plus intacts et peut être due à une aridification du climat. En effet, le sud-Ouest malgache a subi une aridification importante à la fin de I'Holocène, ce qui a causé l'extinction de nombreuses espèces animales et une modification importante de la végétation (Goodman et Jungers 2011). II est possible que cette aridification du climat se poursuive et cause une diminution de la densité des arbustes comme cela a été le cas à Ferlo, au Sénégal (Vincke et al. 2010). De plus, une sécheresse prolongée affecte en premier lieu les individus de régénération, qui périssent (Suresh et al. 2010). Des observations répétées (diversité, structure et régénération) sur au moins deux années différentes doivent être menées pour mettre en évidence les effets de la variabilité interannuelle des précipitations (Randriamalala et al. 2016). En parallèle une analyse de la tendance à long terme (40-50 ans) des précipitations et de leur variabilité interannuelle doit être faite pour démontrer une éventuelle aridification du climat (Randriamalala et al. 2016).

L'inhibition de la reproduction végétale par l'extinction progressive et/ou la baisse de la densité des insectes pollinisateurs peut également être avancée pour expliquer cette mauvaise régénération des fourrés xérophiles. En effet, le site d'étude est une zone de densation des criquets migrateurs (Duranton et al. 2009). De ce fait, une quantité importante d'insecticides y a été déversée au cours des dernières années. Ces insecticides ont également pu tuer les insectes pollinisateurs (Devine et Furlong 2007), d'où une baisse de pollinisation des plantes entomophiles et une production réduite et une faible régénération par germination de graines. Des comparaisons entre (i) les régénérations naturelles et (ii) la diversité et la densité des communautés d'insectes de sites infectés par les insecticides (le site d'étude) et de sites témoins peuvent être faites pour tester cette thèse. Le site témoin pourrait être un site du parc national de Tsimanampesotse, à environ $100 \mathrm{~km}$ au sud du site d'étude. En effet, il est interdit d'épandre des insecticides dans les aires protégées.

\section{CONCLUSION}

La production de charbon de bois affecte négativement les fourrés xérophiles et n'est, par conséquent, pas une pratique durable. Cette dernière réduit leur densité, biomasse ligneuse (dhp) et leur hauteur maximale et affecte la composition floristique. Une disparition localisée, dans les sites de production de charbon de bois, des espèces à bois dur propice à la fabrication de charbon de bois peut survenir dans un futur proche ( $<20$ ans, Ramaroson 2014). De plus, les fourrés xérophiles se régénèrent mal, même si cela n'est pas imputable au charbon de bois. L'aridification du climat et l'usage d'insecticide peuvent être à l'origine de cette mauvaise régénération. Des observations pluriannuelles, l'analyse de la distribution des précipitations au cours des 40-50 dernières années et la comparaison des potentiels de régénération des fourrés xérophiles dans des sites contaminés par des insecticides et dans des site témoins doivent être menées pour identifier les origines de la mauvaise régénération de cette formation végétale exceptionnelle.

\section{REMERCIEMENTS}

Nous remercions le CDE de I'Université de Berne qui a financé ce travail par l'intermédiaire du projet ESAPP Q925.

\section{RÉFÉRENCES}

Ali, A., Xu, M., Zhao, Y., Zhang, Q., Zhou, L., Yang, X. \& Yan, E. 2014. Allometric biomass equations for shrub and small tree species in subtropical China. Silva Fennica 49, 4: \#1275. (doi:10.14214/sf.1275)

Blanc-Pamard, C., Milleville, P., Grouzis, M., Lasry, F. et Razanaka, S. 2005. Une alliance de disciplines sur une question environnementale: la déforestation en forêt de Mikea (Sud-Ouest de Madagascar). Nature Sciences Sociétés 13, 1: 7-20. (doi:10.1051/nss:2005002)

Briske, D. D., Fuhlendorf, S. D. \& Smeins, F. E. 2003. Vegetation dynamics on rangelands: a critique of the current paradigms. Journal of Applied Ecology 40, 4 : 601-614. (doi:10.1046/j.1365-2664.2003.00837.x)

Casse, T., Milhøj, A., Ranaivoson, S. \& Randriamanarivo, J. R. 2004. Causes of deforestation in southwestern Madagascar: what do we know? Forest Policy and Economics 6, 1: 33-48. (doi:10.1016/S1389-9341(02)00084-9)

Chidumayo, E. N. \& Gumbo, D. J. 2013. The environmental impacts of charcoal production in tropical ecosystems of the world: A synthesis. Energy for Sustainable Development 17, 2: 86-94. (doi:10.1016/j.esd.2012.07.004) 
Colón, S. M. \& Lugo, A. E. 2006. Recovery of a subtropical dry forest after abandonment of different land uses. Biotropica 38, 3: 354-364. (doi:10.1111/j. 1744-7429.2006.00159.x)

Delucchi, K. L. \& Bostrom, A. 2004. Methods for analysis of skewed data distributions in psychiatric clinical studies: working with many zero values. American Journal of Psychiatry 161, 7: 1159-1168. (doi:10.1176/appi.ajp. 161.7.1159)

Devine, G. J. \& Furlong, M. J. 2007. Insecticide use: Contexts and ecological consequences. Agriculture and Human Values 24, 3: 281-306. (doi:10.1007/ s10460-007-9067-z)

Duranton, J. F., Franc A., Luong-Skovmand, M. H. et Rachadi, T. 2009. Manuel de Lutte Préventive Antiacridienne à Madagascar à I'Usage des Prospecteurs. CIRAD, Montpellier, France.

Ernest, S. K. M. \& Brown, J. H. 2001. Homeostasis and compensation: the role of species and resources in ecosystem stability. Ecology 82, 8: 2118-2132. (doi: 10.1890/0012-9658(2001)082[2118:HACTRO]2.0.CO;2)

Gardner, C. J., Gabriel, F. U. L., St. John F. A. V. \& Davies, Z. G. 2015. Changing livelihoods and protected area management: a case study of charcoal production in south-west Madagascar. Oryx 50, 3: 495-505. (doi:10.1017/ S0030605315000071)

Goodman, S. M. et Jungers, W. L. 2013. Les Animaux et Écosystèmes de I'Holocène Disparus de Madagascar. Association Vahatra. Antananarivo, Madagascar

Kouami, K., Yaoni, N. \& Honan, A. 2009. Impact of charcoal production on woody plant species in West Africa. A case study in Togo. Scientific Research and Essay 4, 9 : 881-893. Available at <http://www.academicjournals.org/journal/ SRE/article-full-text-pdf/C31F6B716278>

Lachenbruch, P. A. 2001. Comparisons of two-part models with competitors. Statistics in Medicine 20, 8: 1215-1234. (doi:10.1002/sim.790)

Masezamana, H. N., Andrianarisoa, J. H., Raoliarivelo, L. I. B. et Randriamalala, R. J. 2013. Identification et Analyse d'Activités Alternatives à la Fabrication de Charbon de Bois dans le District de Toliara II. Q096 Project Final Report, DERAD, Eastern and Southern Africa Partnership Program, Antananarivo.

Minten, B., Sander, K. \& Stifel, D. 2013. Forest management and economic rents: Evidence from the charcoal trade in Madagascar. Energy for Sustainable Development 17, 2: 106-115. (doi:10.1016/j.esd.2012.08.004)

Navar J., Méndez E., Nájera A., Graciano J., Dale V. \& Parresol B. 2004. Biomass equations for shrub species of Tamaulipan thornscrub of North-eastern Mexico. Journal of Arid Environments 59, 4: 657-674. (doi:10.1016/j.jaridenv. 2004.02.010)

Quesada, M., Sanchez-Azofeifa, G. A., Alvarez-Anorve, M., Stoner, K. E., Avila-Cabadilla, L., et al. 2009. Succession and management of tropical dry forests in the Americas: Review and new perspectives. Forest Ecology and Management 258, 6: 1014-1024. (doi:10.1016/j.foreco.2009.06.023)

Rabeniala, R., Masezamana, H. N., Raoliarivelo, L. I. B. et Randriamalala, R. J. 2013 Clôture en bois dans la commune urbaine de Toliara, une autre source importante de déforestation dans le district de Toliara II. Q925 project final report, DERAD, Eastern and Southern Africa Partnership Program, Antananarivo. Available at <http://www.fao.org/fsnforum/cfs-hlpe/sites/cfs-hlpe/ files/resources/Q925\%20Rapp_Final.pdf>

Rabeniala, R., Raoliarivelo, L. I. B., Masezamana, H. N., Andrianarisoa, J. H. et Randriamalala, R. J. 2009. Gestion de pâturage pour le cheptel de petits ruminants (ovins et caprins) dans une zone semi-aride du district de Toliara II. Q906 project final report, DERAD, Eastern and Southern Africa Partnership Program, Antananarivo. Available at <goo.gl/jS253T>

Radosy, H. O. 2013. Résilience des fourrés xérophiles face au pâturage des petits ruminants et à la fabrication de charbon de bois dans la commune Rurale de Soalara-Sud (District de Toliara II, Région Atsimo-Andrefana). Mémoire DEA, ESSA-Forêts, Université d'Antananarivo, Madagascar.

Ramaroson, R. T. 2014. Durabilité écologique de la production de charbon de bois dans le Sud Ouest malagasy, cas des fourrés xérophiles de la commune de Soalara-Sud (Toliara II, Atsimo Andrefana). Mémoire DEA, ESSA-Forêts, Université d'Antananarivo, Madagascar.
Randriamalala, J. R., Radosy, H. O., Razanaka, S., Randriambanona, H. \& Hervé, D. 2016. Effects of goat grazing and woody charcoal production on xerophytic thickets of southwestern Madagascar. Journal of Arid Environments 128: 6572. (doi:10.1016/j.jaridenv.2016.01.002)

Randriamalala, R. J., Ramananantoandro, T. et Radosy, H. 2015. Productivité en biomasse ligneuse des fourrés xérophiles. In : Transitions Agraires au Sud de Madagascar. Résilience et Viabilité, Deux Facettes de la Conservation. D. Hervé, S. Razanaka, S. Rakotondraompiana, F., Rafamantanantsoa et S. Carrière (eds.), pp 139-145. Actes du Séminaire de Synthèse du Projet FPPSM, 10-11 juin 2013, Antananarivo, IRD-SCAC/PARRUR, Ed. MYE.

Randriarimalala, J. 2016. Dynamiques post-culturales des Fourrés Xérophiles du plateau de Belomotse, Toliara II, Atsimo Andrefana. Mémoire Master 2, ESSAForêts, Université d'Antananarivo, Madagascar.

Raoliarivelo, L. I. B., Rabeniala, R., Masezamana, H. N., Andrianarisoa, J. H. et Randriamalala, R. J. 2010. Impact de la Fabrication de Charbon de Bois sur la Production et la Disponibilité Fourragère de Pâturage en Zone Subaride, Cas de la Commune de Soalara-Sud, Toliara II. Q909 project final report, DERAD, Eastern and Southern Africa Partnership Program, Antananarivo. Available at <http://boris.unibe.ch/69311/1/Q909_rapport_final_product.pdf>

Sander, K., Gros, C. \& Christian Peter, C. 2013. Enabling reforms: Analyzing the political economy of the charcoal sector in Tanzania. Energy for Sustainable Development 17, 2: 116-126. (doi:10.1016/j.esd.2012.11.005)

Seddon, N., Butchart, S., Tobias, J., Yount, J. W., Ramanampanonjy, J. R. \& Randrianizahana, H. 2000. Conservation issues and priorities in the Mikea forest of south-west Madagascar. Oryx 34, 4: 287-304. (doi:10.1046/j.13653008.2000.00134.x)

Suresh, H. S., Dattaraja, H. S. \& Sukumar, R. 2010. Relationship between annual rainfall and tree mortality in a tropical dry forest: results of a 19-year study at Mudumalai, southern India. Forest Ecology and Management 259, 4: 762769. (doi:10.1016/j.foreco.2009.09.025)

Vincke, C., Diédhiou I. \& Grouzis, M. 2010. Long term dynamics and structure of woody vegetation in the Ferlo (Senegal). Journal of Arid Environment 74, 2: 268-276. (doi:10.1016/j.jaridenv.2009.08.006)

Zulu, L. C. 2010. The forbidden fuel: Charcoal, urban woodfuel demand and supply dynamics, community forest management and woodfuel policy in Malawi. Energy Policy 38, 7: 3717-3730. (doi:10.1016/j.enpol.2010.02.050)

\section{SUPPLEMENTARY MATERIAL}

Available online only

Masezamana, H. N., Andrianarisoa, J. H., Raoliarivelo, L. I. B. et Randriamalala, R. J. 2013. Identification et Analyse d'Activités Alternatives à la Fabrication de Charbon de Bois dans le District de Toliara II. Q096 Project Final Report, DERAD, Eastern and Southern Africa Partnership Program, Antananarivo. 